\title{
Bienes Comunes Naturales, contribuciones teóricas a su análisis
}

\author{
NATURAL COMMON GOODS, THEORETICAL CONTRIBUTION TO THEIR ANALYSIS
}

\author{
Micciarelli, G.* Goñi Mazzitelli, A. **1
}

Recibido: 11/11/2019 Aceptado: 20/04/2020

\section{Introducción}

Los conflictos, las disputas y los vínculos entre Ambiente y Economía son una de las principales problemáticas en los territorios latinoamericanos, por la forma en la que repercuten en la reproducción de la vida de sus ecosistemas y comunidades. La pérdida de control y participación en las decisiones que afectan el propio hábitat interpela a la ciencia desde todos sus campos de producción y acción sobre la necesidad de reforzar una teoría sólida que logre consensos en la identificación de sus bienes comunes naturales y proponga formas emergentes y alternativas en su preservación y gestión.

Por un lado, detallados estudios emergen sobre la calidad del aire, del agua, del suelo, que conectan directamente los factores de contaminación con las actividades productivas que gobiernos de distinto signo (derecha/izquierda, conservadores/progresistas) y orientación económica (neoliberal/ neodesarrollista) impulsan en el continente. Por otro lado, como señala el antropólogo Ulrich Beck (2010), el hecho de que los países del Sur acepten los peligros, no debe equipararse a que su población esté conforme, sino más bien a un silencio alimentado por la necesidad. Estos países, ofrecen como su "riqueza" el recurso, limitado en el mundo, del silencio y pasan así a ser los más afectados. De esta manera, la producción del riesgo de la contaminación asociado a una actividad económica a la que no parece haber alternativa, y las posibilidades de sufrir sus consecuencias, son dos hechos que se ven separados en el tiempo y en el espacio. Se mire como se mire, nos dice Beck (2010), se trata de una flagrante injusticia, aunque al tiempo parezcan "catástrofes naturales".

La teoría de los bienes comunes nace del reconocimiento de una pérdida de sentido de la relación hombrenaturaleza, donde la ciencia es también responsable de observar las consecuencias y la destrucción en la relación de este binomio. La "tragedia (de los comunes)" descripta por Garrett Hardin (1998), toma como base la incapacidad de los hombres de gestionar colectivamente los recursos sin tener en cuenta que la ganancia y la competencia, debida a la escasez, produce la destrucción de los mismos.

Si buscamos desde distintos campos de la producción científica un aporte significativo a este debate, encontramos como novedad el surgimiento y desarrollo cada vez más fuerte del concepto de co-gestión de bienes comunes naturales. En primer lugar, porque parte de investigaciones que compilan y analizan casos de estudio en lugares muy distintos del mundo, como los de Elinor Ostrom (1990-2005), premio Nobel de Economía. Los mismos demuestran la eficacia de la conservación de los recursos naturales y sus ecosistemas, a través de una gestión que implica poner en discusión el concepto de propiedad privada, con una serie de acuerdos que parten de la constatación de la importancia de mantener equilibrios entre el consumo y las comunidades que utilizan ese recurso. Al interno de este concepto encontramos el control social implícito en el uso colectivo, con sanciones que no siempre son jurídicas pero que pesan eficazmente en los comportamientos. Por tanto, se pone de manifiesto la necesidad de observar y analizar prácticas tradicionales, antes de querer inventar formas nuevas, que han dado

Universitá di Salerno, Italia.

Centro Universitario Regional Este, UDELAR.

Tekoporá ${ }^{\circledR}$. Centro Universitario de la Región Este. Universidad de la República

(C) Micciarelli y Goñi (2020)

Este es un artículo de Acceso Abierto distribuido bajo licencia Creative Commons (CC BY NC 4.0) 
buenos resultados y que generalmente involucran formas muy diversas a la tenencia o posesión del bien.

En segundo lugar, como señala Boaventura de Sousa Santos (2018), existe un sin número de experiencias en América Latina, partiendo de las resistencias indígenas, de la diáspora africana, de los feminismos, de las culturas campesinas o de la pesca artesanal, que han preservado prácticas y saberes populares o tradicionales, reconceptualizándose en el tiempo y constituyendo ejemplos de alternativas interesantes. Una nueva ontología en la que, sin romanticismos, comprendemos finalmente los mensajes profundos y la tenacidad de estos pueblos al advertirnos cómo las formas de extractivismos prolongados y devastadores están ganando terreno y monopolizando los recursos naturales en el continente (Viveiros de Castro, 2013).

La relación entre recursos naturales y los sujetos que los administran no se agota en el discurso sobre el libre acceso, porque es parte de un sistema relacional profundamente influenciado tanto por la tipología de los bienes, como de las motivaciones que determinan las acciones de explotación por una pluralidad de intereses de difícil simplificación. Lo que aquí proponemos es cambiar la mirada clásica sobre este tema, generalmente existen disputas y negociaciones monetarias que llegan a formas de acuerdos de gestión satisfaciendo a los intereses en cuestión; si los recursos están en riesgo grave pueden protegerse a través de la propiedad pública, si los recursos son plausibles de entrar en una negociación, serán cedidos entonces a la propiedad privada. En este artículo proponemos pensar en una tercera categoría, que es lo común, y por lo tanto comenzar a definir "bienes comunes".

Entonces, si la voluntad política fuera, por ejemplo, la de tutelar la biodiversidad del continente y la cultura de las poblaciones indígenas de la selva Amazónica, una medida drástica adecuada a este fin podría ser declararlo como cierto tipo de "enclave total"; es decir, un espacio protegido en el que nada ni nadie pudiera entrar y salir, dañando el sistema. Sin embargo, hemos visto que ni siquiera en los momentos de auge del discurso del Buen Vivir, fue posible implementar estás opciones radicales, al no tener estudiadas, conceptualizadas y afianzadas alternativas de uso y co-gestión de los bienes naturales presentes al interno de otras economías y otros sistemas de gobierno.

\section{El paradigma de la interdependencia en el "estado de la naturaleza".}

Una de las afirmaciones más conocidas sobre los bienes comunes es que están ubicados detrás del estado y el mercado (Ostrom 2010). En este sentido, el espacio de los bienes comunes es el de la acción colectiva de los sujetos auto-organizados, que pertenece a una esfera social distinta, pero no separada herméticamente, de la de las autoridades públicas y los actores privados que explotan los recursos de acuerdo con criterios orientados a las ganancias. El estado y el mercado no se evaporan, dejando pacíficamente un "espacio en otro lugar" para los bienes comunes. El nudo teórico es, por lo tanto, cómo la auto-organización social interactúa con esos dos polos. En otras palabras, este es el tema de la relación entre lo público, lo privado y lo común. Necesitamos abordar esta dialéctica para no evadir el nudo político que los bienes comunes ponen en evidencia, es decir, si representan una palanca para enfrentar o evadir el problema de la sostenibilidad ecológica y moral del sistema capitalista.

En esta atmósfera con posiciones no siempre claramente definidas, los bienes comunes también resaltan algo más profundo, uno de los nudos cruciales de la política: la interdependencia. A través del paradigma de la interdependencia podemos ver la dimensión política en su esencia, pero debemos entender qué significa "interdependencia", es decir, entre "quién" y "cómo" debe pensarse.

Hannah Arendt, interpretando el problema de la pluralidad y de la acción en común, ha sostenido que no el hombre, sino los hombres habitan la tierra. En cualquier lugar donde se reúnan los hombres, se crea un espacio que al mismo tiempo los une y los separa (Arendt, 1997, p. 18). Esto significa que "la política nace en el inter, y se afianza como relación. Hobbes esto lo había entendido" (p. 7). Este inter en dónde se desarrollan todas las vicisitudes humanas es el "mundo": su existencia hace posible el desafío de la política, es decir esa libertad que cada uno puede conquistar solo en el espacio en el que tiene relación con los otros. Esto explica porque la polis es para Arendt el arquetipo mejor del auténtico espacio público, y explica porque la política no debe ser entendida como el fulcro de la “ preocupación por el hombre", sino por el mundo común, es decir por aquel inter que hace posible a los hombres entrar en relación los unos con los otros, en la única dimensión en la cuál pueden permanecer verdaderamente libres, permaneciendo unidos. Un espacio de este tipo es de un valor inestimado porque es el único que puede no 
solo permitir la libertad, sino también brindar el antídoto a la soledad, que ha transformado en épocas no tan lejanas, a los hombres en parte de la máquina autoritaria (Arendt, 1997).

Si queremos entender este mundo común en su dimensión relacional, debemos tal vez, extenderlo a sus dimensiones materiales, ecosistémicas y naturales. Esto significa colocar la dimensión de la política en un inter que no solo refiera a los seres humanos. Esta es una lección fundamental que responde a la pregunta "qué es la política", pero debemos ir un paso más allá, porque colocar la dimensión de la política en el mundo no significa hacerlo solo entre los seres humanos. De hecho, la interdependencia no solo puede ser la de la pluralidad humana, es más complicado que eso, porque concierne a esta pluralidad en relación con todo el sistema-mundo: los otros seres vivos y el delicado equilibrio entre ellos y el planeta. Es aquí donde los bienes comunes nos ayudan a comprender la política, porque representan lugares donde la interdependencia se mide de forma compleja: entre los seres humanos y entre ellos y la naturaleza.

Después de todo, incluso si la interdependencia no se asume generalmente como uno de los paradigmas principales de la política como se propone aquí, algo que recuerda el concepto, está presente en cada pensamiento clásico. Tomemos, por ejemplo, a los grandes pensadores del contractualismo de la modernidad europea, como Hobbes, Locke y Rousseau, así como a los eruditos que han intentado volver a interrogar sobre la "sociable insociabilidad" que caracteriza a la especie humana (Kant, 2006). Como es sabido, tales visiones hipotizan solo una parte del comportamiento humano, convirtiéndola a veces en una abstracción teórica, un experimento mental, mientras que otras veces la presentan a través de estratagemas pomposos, como por ejemplo con el "velo de la ignorancia" (Rawls, 1999). De esta forma, siempre existe el riesgo de una especie de reduccionismo de las especies, en el cual, para comprender una "verdadera naturaleza humana", se elige un aspecto por encima de todos los demás: interés, racionalidad, justicia, deseo de reconocimiento, etc.

¿Es posible otra forma, incluso si no combina la perspectiva "ejemplar", de ver dinámicas similares en el escenario en el que los seres humanos se organizan antes del salto político que representa el nacimiento del estado? ¿Es posible investigar estos comportamientos en su complejidad y naturaleza, diferenciados y gobernados por contingencias, otras actitudes y elecciones contingentes y en evolución, sin pretender identificar ni la naturaleza del hombre ni su interés o su verdadera razón?

Si estuviéramos buscando una respuesta en un modelo ideal alternativo, terminaríamos en los mismos callejones sin salida, sin poder agregar nada a la capacidad que han tenido autores similares para investigar el alma humana. Si, en cambio, estuviéramos buscando una forma prepolítica similar en la realidad histórica, tendríamos que pedir ayuda a los estudios antropológicos, que de hecho representan un recurso precioso en los estudios contemporáneos sobre bienes comunes².

El camino que sugerimos es más modesto, y es el de la especulación filosófica política y jurídica aplicada a algo que se asemeja mucho a ese modelo del estado de la naturaleza, esta también es una elucubración teórica, pero en una forma atenuada y concreta. Si no pretendemos mirar completamente fuera de la sociedad ya formada y sus instituciones, sino alrededor de sus bordes, entonces podemos encontrar espacios que están al margen de la ley y las formas políticas ordinarias contemporáneas y que nos muestren algo distinto: este es el espacio de los bienes comunes.

Aquellos a los que nos referimos, y a los que se refieren los principales estudios sobre el tema, se basan en el motor que podemos definir, en general, de auto-organización, si bien desde perspectivas muy diferentes, y a veces en neto contraste (ex multis cfr. Ostrom, 1990; Dardot y Laval, 2014; Negri y Hardt, 2017; Kioupkiolis, 2019). Estos son recursos en los cuales la acción colectiva produce procesos de neoinstitucionalización, cuestionando los dilemas de cómo manejar un recurso escaso de manera virtuosa. Las formas son virtuosas no solo si conservan el recurso, sino también si distribuyen su utilidad de una manera aceptada por los miembros de la comunidad, los commoners. Los bienes comunes vistos como pequeños microcosmos en los que la comunidad, o el "hacer común", representan un

2 Un proceso ejemplar que conjuga teoría política con una investigación en el campo es el proyecto, "Heteropolitics", financiado con los fondos ERC-COG-2016-724692, dirigido por Alexandros Kioupkiolis, https://heteropolitics.net/ 
espejo de la política, entendida como interdependencia (Micciarelli, 2018).

De esta manera, los dilemas de una comunidad que se organiza para administrar y usar un bien común nos hablan de algo mucho más profundo (Mark Weber, Koppelman y Messick, 2004). La práctica concreta nos permite anclarnos a la realidad sin tener que sacrificarla; en palabras de Pierre Rosanvallon: "la autogestión no descansa en una antropología como:" el hombre es naturalmente bueno (Rousseau) o el hombre es naturalmente malo (Hobbes). Ésta es ajena a todo moralismo, es hija de la antropología contemporánea" (Rosanvallon, 1976, p. 70).

Aquí, los bienes comunes representan un formidable prisma filosófico para investigar la socialidad humana, y su relación de interdependencia entre si misma y la naturaleza, colocando en el centro espacios de una antropología viva, ambivalente y compleja, en la que la necesidad de autoorganización plantea diariamente los problemas fundamentales de la política.

\section{Lo común, al límite de lo público y lo privado}

Ahora podemos decir con mayor claridad lo que significa que los bienes comunes se encuentren detrás del estado y el mercado: se hallan al borde de los sistemas institucionales y reguladores de esos dos mundos. Este espacio del común está ubicado al límite de lo público y lo privado. Un espacio que es a la vez político, económico y ético, en el que se desarrollan formas organizativas que, por su propia existencia, representan una crítica de la misma dicotomía público-privada, porque identifica sus límites estructurales. Las limitaciones del sector privado son evidentes y muestran el fracaso del modelo de Hardin. Puede ser útil volver a traer sus teorías aquí para reflexionar juntos.

Hardin imagina y reflexiona teóricamente sobre una pradera abierta en un campo no trazado por fronteras privadas, en la cual los pastores pueden traer libremente sus ovejas; por lo tanto, todos tienen acceso libre y durante mucho tiempo no hay problemas. No porque haya surgido un orden espontáneo, sino porque el recurso es suficiente para una serie de pastores que el hambre, las guerras y las enfermedades mantienen constantemente bajo. Pero en algún momento llega el "día del juicio final", que paradójicamente no es una catástrofe, sino el bienestar deseado por todos, esto consecuentemente tiene efectos secundarios en el aumento de los pastores y con ellos también en el número de ovejas. La elección racional a la que se enfrenta cada pastor está comenzando a tomar tonos oscuros: es necesario evaluar si es económicamente conveniente agregar una oveja adicional al ganado, analizando los costos y beneficios de la operación. Dado que la utilidad positiva (el producto de la cría de un nuevo animal) será individual, mientras que la utilidad negativa (que hace que la pradera sea más concurrida y menos productiva) se dividirá por muchos, cada pastor racional solo tendrá que decidir traer una nueva oveja a la pradera, y luego otra y otra. Este razonamiento conducirá inevitablemente a la tragedia de los bienes comunes: la destrucción de la pradera para todos. La privatización y la división de la pradera en lotes se convertiría en una herramienta para revertir el cálculo del hombre racional, que se encargará de no dañar su porción de pradera, claramente delimitada, ya que provocaría la pérdida de productividad y cualquier daño a la propiedad. Los límites son la forma más pragmática que le permitirá seguir usando la tierra y pasarla a las generaciones futuras, que en este caso significa a la propia descendencia (Hardin, 1998).

Aunque Hardin, en su artículo, quería discutir las consecuencias del aumento incontrolable de la población mundial en la tierra y no hacer un tratado sobre cercamientos (enclosures), este ejemplo transmitió un argumento poderoso contra el libre acceso a favor de la propiedad privada. Pero como en todas las parábolas, cada misionero puede contar (y tratar de imponer) su propia moraleja. Entonces se podría sugerir que el comienzo de la ruina ocurre cuando los pastores pobres se ven obligados a aumentar exponencialmente el número de ganado, para hacer frente a la competencia, porque es el mercado para el ganado y sus derivados, y no para el pastoreo, el que presente una falta de regulación. Los efectos son entonces, una lenta crisis de sobreproducción y la caída tendencial de la tasa de ganancia. Por eso hay un desequilibrio, resultante del aumento incontrolado de las ovejas y una disminución en el valor de cada unidad. Podríamos seguir discutiendo, pero sería más útil evitar la tentación de responder a Hardin, y proyectar otra escena en ese campo, cual tela que describe la forma de organizar a los pastores más afines a otra ideología. La narración de una contra-historia, en la cual los pastores se organizan, en un orden comunitario responsable y acogedor, también para los recién llegados, pero especialmente para toda la aldea, sería la alternativa. Cabe señalar que innumerables ejemplos muestran la factibilidad y los beneficios de esta posibilidad, cuya realización concreta ha sido ocultada por una gran parte de la historia económica oficial. Pero el punto es que la 
historia descrita es una historia que vive resultados diferentes en infinitos universos locales paralelos, y como lo enseña la mejor literatura de ciencia ficción, en cada una de estas realidades los protagonistas podrían haber cambiado su destino solo cambiando poquísimos factores.

Lo que debemos entender, por lo tanto, no es la verdad o la falsedad de una historia que no puede responder a ninguna ley universal, sino la función de verificación que cumple. Es decir, si produce un orden del discurso que aspira a legitimar un cierto tipo de orden social. En este caso, el relato de Hardin es realmente similar a la descripción del estado de naturaleza de los contractualistas del ' 600 . Incluso cuando uno no se entretiene en lecturas arcaicas a menudo superficialmente idealizadas, toda la investigación -histórica, económica o sociológicaque logra mostrar esta otra forma de ser de la humanidad tiene, comprensiblemente, un gran éxito, porque demuestra que el estado elemental de la agregación de individuos no es solo la del orden inestable y prevaricatorio del homo homini lupus (Hobbes, 2017).

En un artículo publicado para celebrar el trigésimo aniversario de su famosa publicación, Hardin respondiendo precisamente a las críticas que lo acusaban de haber postulado una falsa equivalencia entre el bien común y el bien no regulado, confirma y aclara su tesis: «un bien común regulado puede ocultar tanto el socialismo como un sistema basado en la libertad de empresa. Ambos pueden funcionar o fallar: "el diablo está en los detalles". Pero en un bien común no regulado no se puede nunca olvidar la mano del diablo: cuando el uso excesivo de los recursos reduce la capacidad del bien, la ruina es inevitable» (Hardin, 1998, pp. 682-283). El problema que plantea es muy grave, pero el desafío de conectar los intereses de todos, con los intereses de cada uno es el nudo político no resuelto que no permite atajos fáciles, porque ambos modelos han fallado: el hecho que los actores del libre mercado, por irresponsabilidad o por lucro, puedan beneficiarse a corto plazo de la sobreexplotación o destrucción de los recursos que administran, incluso cuando son fundamentales para la supervivencia de la humanidad, es en definitiva demasiado evidente (Mattei y Nader, 2010).

La mentalidad propietaria propuesta por Hardin se ha expandido, y se corre el riesgo que continue contaminando muchos estudios sobre bienes comunes, que proponen un abuso de los instrumentos legales de carácter privatizador, que transforman los bienes comunes en nuevas formas de delimitación que se asemejan a las reservas indígenas. 0 por el contrario a formas de autonomia comunitaria regresivas, que aún no queriendo serlo, proponen modelos de organización comunitarios opuestos pero muy similares a la comunidades cerradas (gated communities) con derivas racistas y clasistas, que se están difundiendo en muchas partes del mundo (Day, 2005).

Pero los bienes comunes también subrayan los límites público-estatal, mostrando en forma flexible dónde ellos no pueden, y no deben llegar: ya sea porque son bienes demasiado "grandes", incontenibles dentro de las fronteras de las autoridades nacionales debido a la extensión; sea por el valor ecosistémico universal que representan (se piensa en la selva amazónica, un río que cruza diferentes naciones, una vacuna que la humanidad necesita encontrar con urgencia ...) $)^{3}$; b bien porque, al contrario, son bienes "demasiado pequeños", inextricablemente vinculados al uso y gestión directa, en algunos casos definidos como originarios, de comunidades de habitantes en partes del territorio.

La estrategia es entonces identificar un espacio de lo común que no contrasta con lo público, sino que fortalece su sentido, invirtiendo la lógica, de arriba hacia abajo. La participación popular es la clave para quebrar la dimensión autoritaria que el nivel decisional del Estado normalmente impone. Por otro lado es una estrategia para mostrar otras dimensiones del significado que la palabra "público" debe asumir. Esta consideración es particularmente importante para los bienes comunes naturales, que son los primeros en ser destruidos si proponemos regularizaciones que borran aquellos débiles límites que solo en el derecho público, podemos encontrar como los

3 Decir que en este caso la administración pública no debería llegar se refiere a negar que solo un estado pueda hacerlo, no otros organismos públicos. El problema es que esta estrategia, si se afronta con herramientas tradicionales, demuestra ser completamente ineficaz. De hecho, la estrategia adoptada por los tratados ha fracasado claramente, porque es una fractura precisamente contra la soberanía nacional. La hipótesis más coherente sería la de los organismos supranacionales, que, sin embargo, sigue siendo una hipótesis completamente irreal que permanece en el papel de los tratados. (Ferrajoli, 2013) 
únicos detentores concretos de la privatización neoliberal (Marotta, 2013).

Para comprender cuán importante es dar un peso correcto al rol de lo público, podemos analizar los progresos y límites de los estudios juridicos desarrollados en los últimos años sobre los comunes. Por ejemplo, si tomamos el caso de Italia que es uno de los países europeos que más ha desarrollado el tema, encontramos la Comisión Rodotà por los Bienes Comunes instituida en el 2007 para elaborar un proyecto de reforma del código civil. La Comisión final de su trabajo, propuso un proyecto de reforma, bajo la forma de un proyecto de ley delegada, que tenía como objeto "la eliminación de la taxonomía de los bienes públicos basada en la dicotomía del patrimonio estatal / patrimonio no disponible y su reemplazo por una nueva taxonomía vinculada a la ontología y la función económicosocial de los tipos individuales de bienes"5. La nueva tripartición debería haber previsto tres tipos de bienes: públicos, privados y finalmente bienes comunes. Estos últimos se definieron como objetos tangibles o intangibles que expresan niveles de utilidad funcionales para la protección de ciertos derechos humanos e intereses públicos esenciales, más precisamente "las necesidades colectivas correspondientes al ejercicio de los derechos fundamentales"; en consecuencia, "deben ser protegidos y salvaguardados por el sistema legal, también en beneficio de las generaciones futuras". La definición se basó en una unión original entre el análisis económico del derecho y un sistema naturalista aparentemente legal, que proporcionó un cambio real del eje teórico de la calificación legal de los bienes a los regímenes jurídicos, lo que implica una "inversión conceptual con respecto a las tradiciones jurídicas del pasado"6.

Esta línea de interpretación, propuesta por la Comisión Rodotà, encontró problemas y fracturas entre sus creadores, entre otras razones, debido a la confusión entre bienes públicos y bienes comunes. Una duplicación que no solo no detiene, sino que, según algunos (Maddalena, 2019) refuerza la potestad de los privados de poder administrar estos bienes, para la obtención de lucro. Desde esta perspectiva, es más claro por qué no seguimos al menos de manera ortodoxa, la generosa estrategia teórica de definir una taxonomía de bienes comunes basada en una ontología social y naturalista gobernada por el funcionalismo, como la que anclaría los bienes comunes a las utilidades abordadas al cumplimiento de los derechos fundamentales. Por lo tanto, es necesario combinar una lectura que vincule el lenguaje de los derechos con el de las formas de gobierno colectivo, sin sacrificar ninguno de estos dos polos, ambos esenciales para comprender los bienes comunes.

Hacemos la propuesta de pensar en dos tipos de bienes comunes: los "bienes comunes necesarios" y los "bienes comunes emergentes". Bienes comunes necesarios son bienes que deben considerarse comunes porque incluyen aquellos bienes, tangibles e intangibles, cuya utilidad se considera necesariamente funcional para el ejercicio de los derechos fundamentales. En comparación con el enfoque de la Comisión Rodotá, imaginamos que una mejor protección, a menudo ignorada por el sector público, y aún más por el sector privado, debe implementarse a través de un régimen especial de gobernanza participativa de estos bienes.

Esta estrategia también implica la posibilidad de expandir su categoría, siguiendo la expansión deseable de la categoría de derechos fundamentales de los seres humanos. Entonces, bienes comunes necesarios podrían considerarse no solo los bienes naturales (como el agua), sino también bienes artificiales, como las medicinas necesarias (Rodotà, 2012, p. 107; Ferrajoli, 2012, p. 778; Cauduro, 2017). A partir del hecho que, en virtud de este vínculo indisoluble con la dignidad de la persona, su acceso no puede excluirse sobre la base de criterios de disponibilidad económica.

En este caso, algunos aspectos relacionados con su gestión deberán marcarse de manera participativa, a través de

4 La Comisión de Bienes Públicos, presidida por el senador Stefano Rodotà, toma su nombre en cuanto presidente de la misma, quien representaba una figura rara de jurista e intelectual involucrado en innumerables frentes de la vida civil del país. La misma se estableció en el Ministerio de Justicia, por decreto ministerial el 21 de junio de 2007.

5 Los informes abreviados ordenados por la Secretaría Científica del Ministerio de Justicia, Bienes Públicos, Materiales de Trabajo de la Comisión Rodotà, el 15 de febrero de 2008, se utilizarán como referencia para el trabajo de la Comisión. 6 Ibidem 
procedimientos institucionales que involucren a la audiencia de sus usuarios o sus representantes especiales. En particular, nos referimos a algunas de las decisiones más sensibles para la efectividad de estos derechos, como, por ejemplo, las reglas de distribución, concesiones, la asignación de servicios de acuerdo con criterios económicos y de protección. En este caso, nos enfrentamos a una definición del régimen de gobierno en el que las formas de participación popular representan un límite para cualquier acto prodrómico de exclusión realizado por el administrador, tanto público como privado ${ }^{7}$. A los titulares de los derechos de uso también se les debe otorgar una protección inhibitoria generalizada y una legitimidad procesual especial para intervenir en caso de daño a sus derechos.

Luego están otros bienes comunes, capaces de garantizar el cumplimiento de los derechos que enriquecen el catálogo de los bienes fundamentales, en particular en la dirección de los bienes sociales y civiles. Estos son los "bienes comunes emergentes", es decir, esos bienes pueden hacerse comunes cuando viene valorizado el proceso fundamental para la democracia de la formación de una comunidad que los tutela. Es decir que más que cuidar un bien en sí mismo, cuida en forma recíproca las necesidades que ella misma expresa. Esto sucede cuando los bienes son percibidos como propios por parte de una colectividad amplia, pero no en un sentido de propiedad, ni de pertenencia ideal o territorial, sino comunitario.

Los bienes comunes urbanos por ejemplo, son el arquetipo de esta categoría (Stravides, 2014; Micciarelli, 2014), pero en este momento podemos decir que incluso en estos casos, una de las principales fuentes de inspiración no proviene de la ciudad, sino del entorno rural, y esto es lo que ahora debemos considerar para imaginar esta forma de Gobierno alternativo al público y privado.

\section{Radicalizar la "democratización de la democracia"}

Ahora bien, ¿cómo podemos imaginar en forma concreta la participación de los ciudadanos, o habitantes de un territorio en la gestión de estos bienes comunes?

Para comprender lo que significa administrar bienes comunes de una manera que se encuentra al margen de los sectores público y privado, tenemos que profundizar en la dimensión comunitaria y su relación con la tierra. Es sumamente importante observar en la historia como la interdependencia es afrontada por la inteligencia colectiva.

En todas las latitudes hay prácticas y modelos para gestionar recursos comunes, como tierras colectivas, recursos fluviales, montañas. Si miramos cada una de estas historias solo como un ejemplo de cómo podemos explotar eficientemente un recurso, captaríamos solo un pequeño aspecto. Pero si las observamos detenidamente, cada una de estas experiencias representa una formidable sección transversal de la forma de pensar de una sociedad, son el espejo de las relaciones de producción y reproducción. La inteligencia humana ha desarrollado en diferentes mundos formas muy similares, simples y al mismo tiempo complejas de organizar los recursos agrosilvopastorales: comunidades agrícolas, normas y comunidades del arco alpino oriental, usos cívicos, dominios colectivos, participaciones emilianas, ademprivi sardos, montes comunales, veciñais en man común, huertas valencianas y muchos otros podrían agregarse. Nos equivocaríamos si midiéramos el peso de tales experiencias solo en función del número de personas que pueden involucrar. Estas piezas de la historia viva no muestran formas primordiales, como tales superadas por el progreso, sino elementales, como tales constantes en las formas de organización de las cosas comunes.

Una de las características fundamentales de estas experiencias es poner los derechos de gestión comunitaria en igualdad de condiciones con los derechos de uso colectivo. Analizando el desarrollo histórico de la categoría de uso, según Paolo Grossi nos enfrentamos a un fenómeno fáctico, prejurídico que se impone a la ley, ya que "representa la respuesta natural a las necesidades primordiales de supervivencia. Precisamente porque es estimulado por fuerzas irresistibles llamadas hambre, sed, frío, calor, etc., el actus utendi no es una expresión de libertad, sino de necesidad" (Grossi, 1992, p. 175).

\footnotetext{
7 Una forma de responder a la crítica justa desarrollada de que "no existe protección legal (y menos constitucional) contra el Estado que transfiere a la comunidad privada activos (bienes comunes) que no están en propiedad privada" (Mattei, 2011, IV)
} 
Por lo tanto, los derechos de uso parecen ser otro elemento crucial, que debe caminar junto al tema de la gestión comunitaria de los bienes comunes. El derecho de uso nos sirve para superar la idea de que las cosas están formadas por meros objetos: reificarlos va de la mano con la extensión de los derechos de propiedad como paradigma social. Perder de vista los derechos de uso significa absolutizar, con las gafas deformantes del modelo individualista de pertenencia, el vasto mundo de los bienes, borrando sus peculiaridades, funciones, características y razones (Grossi, 2006, pp. 24 y ss.). Esta lectura, ahora parte de la herencia inconsciente del Occidente contemporáneo, se presta para extender la voluntad del hombre de poder sobre todo lo que lo rodea. La catastrófica falacia de este enfoque es aún más evidente cuando consideramos las cosas no como simples objetos, sino incluso como sistemas vitales. Un claro ejemplo es el del agua, los bosques u otros arquetipos de los bienes comunes necesarios; sujetos del contexto ecológico, como tales, que viven, que acogen otras formas de vida y, por lo tanto, no pueden plegarse en instrumentos de seres humanos solamente, y mucho menos una minoría privilegiada.

Hasta ahora hemos descrito la participación como una clave importante para deconstruir el concepto excluyente de propiedad. Pero debemos estar atentos cuando hablamos de participación, porque nos arriesgamos otra vez a que detrás de las promesas se manifiesten derechos ineficientes y grandes desilusiones, en parte porque las políticas participativas son un leitmotiv difundido, al representar un truco para colmar los deficits de legitimación política de las instituciones.

A esto le agregamos que el pluralismo jurídico contemporáneo se alimenta de procesos participativos, que el giro neoliberal tiene como objetivo ampliar y utilizar el déficit en la legitimación de los sistemas democráticos representativos (Jessop, 2002).

Tenemos que pensar entonces a la participación en forma distinta. El discurso sobre los bienes comunes no se limita a la pretensión de garantizar mayores derechos, expectativa generalmente desilusionante, en particular cuando se habla de procesos institucionales y derechos fundamentales. La diferencia relevante es que luchar por los bienes comunes, significa siempre una reivindicación de una forma de gestión diversa de los mismos, que propone su fundamento en los derechos de uso no exclusivo y extendidos a colectividades concretas más amplias de aquellas contempladas en el derecho de propiedad entendido en forma clásica. Estas formas de gobernanza evocan la importancia de la participación cívica, como instrumento para ampliar la base decisional a aquellos para quienes el destino de los bienes comunes es realmente importante para su propia supervivencia.

Retorna aqui el desafío de la interdependencia como desafío de la política, que no se puede resolver proponiendo la palabra democracia, como un "adjetivo" capaz de ajustar por sí solo cada problema: la democracia antes de ser la solución, es aún el problema a resolver. Como plantea Boaventura de Souza Santos, quizás podamos usar el gran debate y las esperanzas, a menudo también las desilusiones, que surgieron en torno a la democratización de la democracia (de Sousa Santos, 2003) para legitimar las formas de uso colectivo y la gestión de los recursos comunes que siempre van de la mano con la gramática de las herramientas participativas.

Podemos citar un ejemplo latinoamericano de involución de los instrumentos participativos, que puede enseñar mucho a la teoría de los bienes comunes. En Brasil, el verdadero antecedente, y la madre del presupuesto participativo, son las redes de vecindarios, que habían constituido algo más que un contexto político, es decir, un humus fértil para el reclamo posterior del presupuesto participativo. Sus prácticas de montaje, lejos de ser un modelo anticuado, de alguna manera reemplazado por formas más bien equipadas, representan en cambio la base sobre la cual se han estimado las formas institucionales del presupuesto participativo; no solo eso, la vitalidad autónoma y la permanencia de los movimientos también depende del buen funcionamiento de los modelos más formalizados de democracia participativa.

Cuando en 1989 el Partido dos Trabalhadores ganó las elecciones municipales, se pagó el precio de una larga ausencia de organismos intermedios tradicionales. Pero la dictadura no pudo borrar la vitalidad democrática, que habían encontrado otras formas de existir y resistir. Por ejemplo, en algunas ciudades donde el MDB (Movimiento Democrático Brasileño) era fuerte, se habían adoptado políticas participativas como instrumentos de presión y pedido de transparencia en los niveles federales y estatales que habían sido controlados por los militares durante la dictadura entre 1964 y 1985. Luego se desarrolló una nueva "ingeniería institucional", con algunos experimentos que fortalecieron canales alternativos de participación en la vida civil. Por ejemplo, el "Forum do Prezeis", creado durante la primera administración de Jarbas Vasconcelos en Recife (1986-1988), que permitió la participación popular en la administración de proyectos de urbanización en "áreas especiales de interés social"; o incluso el "Funaps" comunitario, fondo que la administración de Luiza Erundina en São Paulo (1989-1992) dedicó al apoyo de 
las cooperativas que promueven procesos de autoconstrucción de viviendas (Bairle, 1998). Su arquetipo es mutirão, una forma colectiva de autogestión de los campos, recuperación personal y construcción solidaria de las partes más pobres de la población, que hoy ha adquirido un significado más amplio que las acciones colectivas basadas en el mutualismo (Almeida y Muçouçah, 1991).

Esta es la parte más interesante, y hoy aparentemente derrotada de esa historia, ya que al institucionalizarse y ser casi una competencia por fondos entre distintos grupos locales, el presupuesto participativo perdió sus bases de solidaridad comunitaria, pedido de transparencia al estado y trabajo de planificación deliberativa de sus inicios. Sin profundizar ahora en las razones de su "termidoro", que hizo del presupuesto participativo algo muy diferente de sus orígenes (Bairle, 2003), podemos tomar un punto de partida importante: vincular los derechos de uso y gestión en territorios comunes, que representan parte de una estrategia participativa y reivindicativa de los últimos y de los sin tierra, o de los "sin partes" (Rancière, 1995). Debemos radicalizar esta lección comunitaria de la primera fase de la democracia participativa, imaginando nuevas instituciones y nuevas formas legales que exploten en su beneficio las redes de sistemas reguladores que caracterizan el derecho contemporáneo (Amendola, 2010, p. 175).

Como muestran muchos estudios sobre bienes comunes urbanos, y en particular por el nuevo instituto de uso urbano cívico y colectivo nacido en Italia (Capone, 2016; Micciarelli, 2017; De Tullio, 2020), una buena estrategia es aprovechar la oportunidad de políticas participativas para servir a la lógica comunitaria de bienes comunes urbanos, imaginando una nueva brújula institucional, con dos ejes. El primero consiste en la atribución de nuevos derechos de uso colectivo en partes cada vez mayores del espacio, público y privado. Esto se debe principalmente a una razón política: un derecho de acceso difuso es prodrómico para la realización de una comunidad verdaderamente plural y heterogénea. De esta manera, puede ayudar a eliminar los grumos excluyentes de la lógica de afinidad agregante, al menos en la parte que constituye la red implícita de la postura propietaria, que excluye lo diferente de sí mismo, con métodos y formas muy lejanas a las pacíficas. Extender los derechos de uso colectivo también responde a una segunda necesidad de naturaleza más técnico-política: el paso de un bien público de cualquier propiedad estatal al patrimonio disponible está relacionado con la pérdida, incluso tácita, del destino para el uso público del bien ${ }^{8}$. Esto significa, por el contrario, que es precisamente a través de una extensión de usos que podemos proteger un activo de su venta ${ }^{9}$. Esto también puede ayudarnos a arañar el moloch de la propiedad privada, ayudándonos a atacar lo abandonado por el "no uso", comenzando con la imposición de nuevas servidumbres de uso colectivo.

El segundo polo de nuestra brújula institucional se refiere en cambio a la gestión común, que en los bienes comunes urbanos debe ser directa, coordinada por la comunidad cívica que se ocupa y cuida de ello (Caffentzis, 2012).

La razón de este segundo aspecto encuentra plena consonancia con uno de los objetivos más ambiciosos, y con demasiada frecuencia olvidados, de la democracia participativa: el impulso por el autogobierno. En primer lugar, necesitamos un camino pedagógico, que encuentra en la forma con que se crean estas nuevas instituciones una necesidad inevitable: «el espacio público, como el doméstico interior, se convierte en puro espacio vacío si no está estructurado, articulado, si no se le da cuerpo. Debe tener una forma institucional, no menos que nuestros cuerpos personales complejamente integrados, una forma que no puede existir sin una estructura. Sin forma y articulación no puede haber identidad, definición, no puede tener esas especificidades que producen variedad. Lo que se debate hoy, cuando se trata de instituciones, no es si deberían existir, sino qué forma deberían tener: libertaria o autoritaria» (Bookchin, 2017, p. 449).

Esta utopía no es otra que la memoria antioligárquica que caracteriza el largo proceso de expansión de la democracia, al menos en su interpretación metodológica, pero no por esta razón meramente formalista. Nuevamente, el amanecer de la democracia participativa puede ayudarnos. Esta vez no estamos viendo el caso latinoamericano, sino el de América del Norte. La escena es considerada por muchos como la madre de la democracia participativa: nei campus universitari del Michigan, los "Students for a Democratic Society" (SDS)

8 Cass. Civile Sent. Sez. UN. Sentencia n. 7739 del 25 febbraio 2020.

9 Una lectura que parece confirmar y encajar coherentemente con la propuesta, presentada durante algún tiempo, para aprovechar la analogía entre la propiedad estatal posible y necesaria también para calificar los bienes comunes en los emergentes - posibles y necesarios, (Micciarelli, 2014). 
invitados por el mayor de la "United Auto Workers" (UAW), que mucho antes del '68 era el escenario de asambleas y ocupaciones que pretendían denunciar la imagen de la democracia estadounidense, considerada perfectamente funcionante, pero al alto precio de dejar individuos aislados y una vida política atrofiada (Hayden, 2012) ${ }^{10}$. Pero más que un programa, la convención de Port Huron fue una declaración que brindó la oportunidad de experimentar lo que realmente importaba para sus activistas: no solo el contenido de un documento excelente, sino una práctica de escritura abierta, compartida y plural, desarrollado a través de asambleas y reuniones que duraron meses (Hauben, 1995). Un ejemplo vivo de las prácticas de democracia participativa imaginadas podría proyectarse en la misma sociedad (Miller, 1987, p. 329) ${ }^{11}$. Incluso un fragmento de papel puede realizar la función de materializar un espacio común en el que razonar mediante la acción en común.

En esta agitación, Sherry R. Arnstein publicó un artículo breve pero influyente en el que declaró que hablar sobre la participación ciudadana significaba esencialmente "redistribución del poder que permite a los ciudadanos pobres, actualmente excluidos de los procesos políticos y económicos, ser explícitamente (deliberately) incluidos en el futuro" (Arnstein, 1969, p. 216).

Este ejemplo no sirve claramente a la hora de reivindicar lugares más adelantados o menos en las formas de la democracia participativa en el contexto norteamericano como prueban a hacer algunos. Si hay algo que nos enseñan los bienes comunes es que pueden ser comprendidos sólo en una óptica anticolonialista de las ideas políticas y de las formas jurídicas, que tienen la capacidad de manifestarse diacrónicamente o sincrónicamente en múltiples latitudes. Es decir que debemos observar las varias pequeñas historias desconectadas en tantas partes del mundo para superar nuestros límites y extendernos más allá de las lecturas consolidadas, reivindicando nuevas formas de democracia política, y del uso de los recursos comunes, que han combatido contra las tramas de los oligopolios y de las oligarquías, políticas y económicas. Pero, no sólo podemos recopilar historias antiguas, debemos actualizarlas, aprovechando las oportunidades de los términos contemporáneos: participación y soft law. Los bienes comunes son otra oportunidad que no debe perderse para tratar de redistribuir este poder, aprender a hacer algo aún más difícil que su conquista: aprender a administrarlo.

\section{La planificación territorial en la valorización de los bienes comunes}

En esta fase histórica, los estudios demuestran que es necesaria una transición ecológica que supere los modelos de producción y consumo de masa, que han sido depredadores y con altos impactos negativos para el ambiente, los ecosistemas, los seres humanos y toda la biodiversidad del planeta. Neil Brenner (2014), profesor de planificación territorial de la Harvard Graduate School of Design señala el surgimiento de una "teoría urbana sin afuera". Es decir, una condición emergente de urbanización planetaria donde aún los espacios que permanecían fuera de los tradicionales centros de aglomeración, por ejemplo las rutas mundiales de los barcos de carga, o las rutas de transporte o las infraestructuras de comunicación, los sitios de extracción, los enclaves turísticos, alpinos y de la costa, las zonas agroindustriales, y las reservas "naturales" como los océanos, desiertos, selvas, montañas, tundra, la atmósfera, están siendo englobados en un "paisaje operativo mundial”.

Mientras que los Estados Nacionales están preocupados por la dimensión de Planificación y Ordenamiento Territorial Nacional, los desafíos que deberán afrontar, como señala, Guido Viale (2011) son el cambio climático y la inminente crisis hídrica, las migraciones forzadas por guerras promovidas por la extracción de materias primas y la expulsión forzada de campesinos por la privatización de las multinacionales de las tierras agrícolas y su producción. Para el 2050, más de dos tercios de la población mundial estará bajo "stress hídrico", es decir que con el calentamiento de la tierra, si no se inicia una planificación que prevé un consumo responsable de los recursos naturales se podría llegar a una crisis hídrica y de alimentación a mitad del siglo XXI. Esto se evidencia en recursos como el agua, ya que el $70 \%$ del agua disponible en el mundo es usada para la agricultura, el $20 \%$ para la industria, y solo el $10 \%$ para el consumo doméstico. Esto significa que el $\mathbf{9 0 \%}$ del agua del planeta está bajo la responsabilidad del sector privado (Stockholm International Water Institute, 2012).

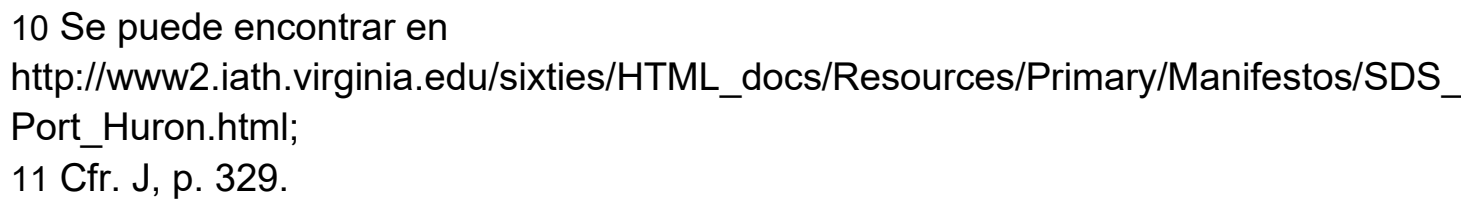


Si consideramos que la Planificación a fines del Siglo XX adopta el concepto de "desarrollo sustentable", nos preguntamos cómo es posible que los resultados sean tan escasos y sobre todo que no haya una percepción cultural difusa del riesgo medioambiental y de la necesidad de un consumo crítico (Allen, 2001). No es casual nos dice Serge Latouche, ya que cuando en el 1973, luego del Environmental Summit de Estocolmo '72, Maurice Strong, responsable del programa de las Naciones Unidas para el Medio Ambiente (PNUMA) inventa la expresión "desarrollo sustentable", no piensa en un desarrollo alternativo al modelo económico de explotación y acumulación de las riquezas, simplemente se hace presente la necesidad de incorporar medidas que preserven los recursos naturales para continuar su explotación. No se hace tampoco referencia en este nuevo desarrollo sustentable a las diferencias en los niveles de contaminación Norte-Sur, que más tarde en el 1997, confirman los expertos con ocasión del tratado de Kyoto. No hay dudas que el modelo económico y cultural prevalente hace que los niveles de consumo crezcan en los países "desarrollados", y por lo tanto crece la contaminación, destruyendo sistemáticamente los diversos ecosistemas mundiales (Latouche, 2007).

Los pilares en los que se basan las teorías alternativas al "crecimiento" y el desarrollo son los de crear una nueva cultura que apunte a: la "reducción" de consumos y de contaminación, la "reconversión" de todas las energías y materiales de la industrialización y de la urbanización, y la "relocalización" de los bienes y productos, volviendo a formas de auto-consumo y producción local (Viale, 2011).

En estas vertiginosas transformaciones los bienes comunes deben encontrar un contexto jurídico- normativo pero también que reúna interdisciplinariamente un estudio del territorio y de su gestión, que permita la valorización y defensa de los mismos para cambiar radicalmente el modelo económico actual. En este sentido la investigación no puede permanecer ajena a promover procesos de planificación colaborativa que apunten a empoderar los grupos locales que se proponen como parte del proceso de valorización o creación de bienes comunes, compartiendo herramientas para intercambiar conocimientos y contribuir a reforzar el rol protagonista de las mismas.

En la actualidad a nivel internacional se afirma esta modalidad en la salvaguarda de los bienes comunes naturales, es decir la posibilidad de planificar y gestionar en forma multiactoral las políticas públicas ambientales y sus consecuentes acciones en el territorio en relación a los servicios ecosistémicos (Mancilla y Bodin, 2019).

Cómo señala Donna Haraway, los procesos que restituyen protagonismo a diversos actores en diversos niveles de gobierno pueden despertar un "sentimiento profundo ecológico", necesario para transformar las prácticas humanas que modifican el ambiente desde un cambio de concepción del lugar que el hombre ocupa en el mundo y de los derechos de la naturaleza que han sido alejados de las concepciones universales contemporáneas (Haraway, 2019).

En las investigaciones sobre procesos participativos y gestión de bienes comunes, se releva una situación ambigua. Al mismo tiempo que se evalúan en forma positiva la creación de Ministerios del Ambiente, planes nacionales, políticas de ordenamiento territorial, y figuras de participación ciudadana en comisiones de cuenca, de gestión de áreas protegidas, por ejemplo, o de presentación en audiencias públicas de planes y programas en la tutela de los mismos. Se identifica por otro lado el actual retraso u omisión que tienen los gobiernos locales y nacionales latinoamericanos, en temas de legislación, de control de actividades privadas, así como de formas de co- gestión multiactoral de los recursos. Por el contrario, como señala Eduardo Gudynas, en el discurso político los gravisimos efectos ambientales que se presentan como resultado del sistema económico extractivista vienen minimizados a favor de los criterios de beneficio y utlidad económica de la naturaleza (Gudynas, 2014).

“ (...) la Naturaleza pierde su organicidad, se la fragmenta, y algunos de esos fragmentos tienen un precio y dueños, convirtiéndose en mercaderías. Una vez transformados en mercancías son ingresados a los mercados. Esta penetración del mercado en la Naturaleza ha sido impresionante. Sus expresiones más conocidas son la difusión de categorías como «capital natural» o «bienes y servicios» ambientales, donde se convierten en mercancías no sólo a seres vivos o recursos inertes, sino que se intenta comercializar hasta las funciones de los ecosistemas." (Gudynas, 2014, p30).

La necesidad de desarrollar un razonamiento a nivel nacional, pero que parta de los contextos locales, es inminente. Se deben identificar nuevas estrategias que propongan un uso y gestión sostenible de los bienes comunes naturales, como alternativa a los desarrollos tradicionales de gran escala, incluso los llamados desarrollos sustentables que no cambian la matriz de intensificación en el uso de los recursos naturales y concentración de la ganancia. 
¿Cómo hacer para que estos modelos económicos que han escapado al control de los territorios, de sus poblaciones originarias, humanas y no humanas y se mueven en ámbitos transnacionales de decisión, vuelvan a esa dimensión de gobernanza colectiva citada anteriormente en este artículo?

Es necesario reconocer los límites de creer que existe una voluntad altruista generalizada que desea realmente una salvaguarda y gestión colectiva de los bienes comunes naturales, cuando en realidad la fractura entre movimientos ambientalistas y otros habitantes crecen vista la contraposición discursiva, muchas veces alimentada por partidos políticos neoliberales y desarrollistas, que proponen estas formas de explotación de los recursos como portadores de beneficios reales económicos en términos de empleos e infraestructuras. Salvo en contados casos, que las comunidades locales ponen al centro propuestas económicas en armonía con la naturaleza, como la agroecología y el turismo responsable, la gran parte de la población visualiza dificultades para una sustitución de las actuales formas de economía por otras más sostenibles. En parte por ello, se planta como central la necesidad de trabajar en una gobernanza ambiental que produzca debates profundos y proponga alternativas económicas creíbles, en esta transición que se vería ampliamente beneficiada si contara con un apoyo decidido y facilitado por los gobiernos nacionales.

Un factor clave, sumamente necesario en el éxito de las acciones en la salvaguarda de los bienes comunes, y en la que los territorios están actualmente lanzando una alerta fuerte y clara, es que no basta con inversiones, acciones, técnicos y recursos para afianzar una política en la temática. Se debe construir una relación de confianza entre el gobierno y los pobladores ya que el exceso de técnica o la toma de decisiones políticas a puertas cerradas (Villasante, 2012), lleva a un claro sentimiento de desconfianza y frustración sobre las posibilidades de la acción colectiva en lo local.

Entre las causas de estas frustraciones, varias de las investigaciones señalan la percepción de los habitantes de la no realización de formas de consulta reales sobre los emprendimientos económicos que se instalarán en los territorios. Mientras que varios grupos organizados demandan estos espacios habilitantes de frente a la fuerte certeza de la degradación del ambiente y la pérdida de los recursos naturales, el temor a perder las inversiones las relega a simples observadores o se les consulta solo al final, en audiencias públicas, cuando los proyectos ya están decididos. Es decir, que en estos momentos la percepción que mayormente se siente en el continente es la de impotencia al interno de un sistema democrático que negocia a puertas cerradas con los grandes capitales, así como de vulnerabilidad al ser dejados solos por el Estado de frente a las consecuencias que traerán en lo local el crecimieto de la contaminación y la escasez de recursos fundamentales por falta de normativas severas en su extracción.

Durante el 2018 se elaboró en Uruguay el Plan Ambiental Nacional para el Desarrollo Sostenible, la Universidad participó activamente para que fuera discutido y enriquecido por aportes de diversos actores en las 19 regiones del país. Una de las solicitudes más importantes fue justamente la necesidad de una mayor presencia del Estado, no solo más Estado, sino un Estado cercano, que coordine en forma inteligente y continua con los recursos humanos en lo local, que escuche y no imponga decisiones "desde arriba” (Goñi, Bisio, Chiglino y Venegas, 2019).

Los habitantes de los territorios son los primeros en tomar conciencia sobre las consecuencias de formas no sostenibles de desarrollo territorial, porque se reflejan en fenómenos notorios en la vida cotidiana y en el deterioro de los servicios eco sistémicos. Por ejemplo, un tema que reiteradamente se pone en el centro de los conflictos en América Latina, es el agua potable, como uno de los más sensibles frente al cuál se manifiesta una falta de confianza en el accionar del Estado. Por un lado los organismos pertinentes realizan controles y aseguran que el agua es potable, por otro, la población ve señales preocupantes, escasez, olores, coloraciones, mortandad de peces, o actitudes poco responsables de los productores alrededor de las tomas de agua. No existe una interacción de colaboración e intercambio, más allá de la denuncia. Es decir que los técnicos estatales, no pueden beneficiarse de una vigilancia local, con la virtud de compartir las responsabilidades del monitoreo, que lleva a una toma de conciencia por parte de la población multiplicando los cuidados y el control social, en las prácticas culturales con respecto a la contaminación de los recursos hídricos. Así como los habitantes, no pueden manifestar sus inquietudes, tampoco pueden acceder a los estudios para verificar la calidad del agua, o transmitir su inconformidad con el estado del agua actual en varias localidades. A esto se suman mecanismos sociales profundos de auto defensa, existe una conciencia extendida de que el recurso hídrico se deteriora, aún existen generaciones que nos relatan cómo tomaban el agua de los ríos, se bañaban sin temor y sabían que debían ser cuidadosos, pero que de los pozos, o 
de las instalaciones del gobierno podían proveerse de agua potable, este conocimiento popular los lleva a enfrentarse al conformismo que les propone el Estado por la degradación en la calidad del agua (Goñi et al., 2019).

La alta participación de las comunidades a los conflictos ambientales está presente en los territorios, en los pueblos pequeños, en la vida de las personas, en forma mucho más presente de lo que el debate público mediático, generalmente producido en las grandes ciudades o capitales, permite apreciar.

El desafío mayor entonces desde la investigación es el de construir acciones que restituyan una confianza mutua, entre gobierno, sociedad civil, empresas y pobladores. Pero para que ello suceda tiene que haber un trabajo intensivo en el que se clarifiquen las prioridades y los criterios con los que se ordenará el territorio. Es decir que se hacen necesarios espacios "habilitantes", en los que se reconozca la pertinencia de manejar informaciones con discrecionalidad, pero que permitan realmente en tiempo y forma reforzar modalidades de planificación multiactoral en los territorios sobre las temáticas prioritarias (Cellamare, Cognetti y Goñi Mazzitelli, 2018). Sin estos espacios al interno de procesos que luego se plasmen en planes, programas y normativas de ordenamiento territorial para el uso de los bienes comunes, no se logrará una planificación deliberativa real. Es decir que necesitamos incentivar a los Estados a mejorar sus procedimientos para que se transformen en verdaderas ocasiones de toma de decisiones informadas y lograr evaluar en la escala de valores y condiciones de vida en lo local, cuáles son los pesos que debe darse a cada transformación, según una evaluación detallada de los costos y beneficios en la economía, el ambiente, la salud, entre otros.

Para llevar adelante este razonamiento no debemos olvidar que los gobiernos no están solos en esta tarea. La antropóloga y urbanista Patsey Healey (1997) nos recuerda que entre los años 80 y 90 comenzaron a elaborarse e implementar los Planes de Desarrollo local. La planificación estratégica fuerte teóricamente era sistemáticamente debilitada por la lobby de las empresas que convencían a los gobiernos nacionales de ignorar las estrategias que estos planes ponían en campo y que tenían como eje central a los gobiernos locales. Una guerra entre los intereses bottom up, y top down caracterizaron la planificación de ese período. Las investigaciones demostraron que los programas fallaban porque por un lado muchos de los actores se lamentaban de la falta de estrategias coherentes $\mathrm{e}$ integradas que tuvieran una dimension espacial declarada.

En particular la lobby de bienes raíces, de la construcción, de las industrias, estaban pidiendo reglas claras para establecer un mercado con valores declarados que disminuyeran los riesgos. Por otro lado las lobbies ambientalistas pedían planes y reglas aún más específicas que preservaran las condiciones naturales sobre la ganancia.

Healey nos habla de una contradicción o de tiempos distintos entre estos distintos intereses, ya que existen dialogos separados entre Estado, Mercado y Sociedad civil, sobre las preocupadas por la calidad ambiental y el desarrollo local. La autora se preguntaba si era posible que la planificación produzca injusticias redistributivas. Varios estudios indicaban que en las áreas donde existían intereses privados, las políticas de planificación traían beneficios porque salvaguardaban la calidad ambiental y social. Mientras que en las áreas donde no existía inversión privada, en general las más pobres, el estado invertía pero en forma sectorial, sin considerar los planes de desarrollo local, por lo tanto la "calidad del lugar" disminuía. El público no atraía las inversiones privadas.

Esto daba lugar a debates entre distintas corrientes, los neoliberales, señalaba Healey, sostenían que el desarrollo local junto a la planificación espacial estratégica no eran adecuados en la era de la globalización y los capitales transnacionales. Por otro lado las corrientes postmodernas que aún tenían dificultad para responder a estas críticas, reconocían que había mucho para explorar y que se debía apuntar a desarrollar intervenciones puntuales creativas. La batalla está presente, desde los inicios de la planificación colaborativa desarrollada en el mundo anglosajón, entre Norteamérica y Reino Unido, por nombres como Forester, Innes, Joch, Harper y Stein, que viven esta contradicción de tener políticas públicas, aún no adaptadas a gobernar la complejidad. Mientras que los grupos de poder estructurados intentaban minar la importancia de estos procesos que demuestran la voluntad de aprender y ponerse en discusión de varios grupos, y la de negociar en forma horizontal para desarrollar proyectos de territorio. Tomando estudios de Giddens y Foucault, Healey recuerda que los procesos de planificación dependen no solo de intereses particulares de los actores, sino también de culturas institucionales arraigadas. Es por esto que identifican la necesidad de romper con los tradicionales procesos de planificación jerárquica y burocrática, a través de la construcción de partenariados, alianzas y redes de sujetos. Pero también producir proyectos tangibles con consecuencias materiales.

En definitiva el Colaborative Planning buscaba la justicia social y espacial en las intervenciones, es decir que beneficien a una amplia gama de actores. Considerar la planificación como un proceso que da varios resultados, no solo el plan; las alianzas, los modelos de desarrollo local, son un resultado por ejemplo, las estrategias de 
implementacion que sirven para un contexto y no para otro; las relaciones entre los distintos niveles de gobiernos, las nuevas reglas y legislaciones que nacen de las exigencias de estos procesos en contacto con sus realidades actuales.

La antropologia cultural nos demuestra que las relaciones sociales significativas no son guiadas solo por una fuerza estructurante. En la construcción de los mundos sociales no entran en juego solo fuerzas económicas, como el debate de los estudios feministas, poscolonialistas y de geografía cultural o capital social demuestran. Operan también fuerzas socio- culturales, políticas y ambientales.

En varios lugares del mundo la planificación se reduce a un control del uso del suelo. Una de las razones es la dificultad en tener recetas idénticas para culturas diversas. Porque la planificación tiene que ver con las formas de organización y gobernanza de una sociedad. El Collaborative Planning pretende considerar esa diversidad. En cada sociedad existe un abordaje diverso del gobierno del territorio, como existe un abordaje diverso al cuidado de los ancianos, a la salud, etc. El rol de la planificación debería ser interrogarse sobre las prácticas de governance existentes en el lugar y de ayudar a estas comunidades a mejorar la calidad de sus ambientes, sobre todo donde están fracasando. En términos de la calidad de vida de las personas de un lugar, lo que importa es el juego recíproco de estas fuerzas y el modo en el que esto incide sobre la habilidad colectiva de obtener beneficios y limitar los impactos negativos (Healey 1997). El paradigma de derechos de la naturaleza reciente, cambia en parte el análisis institucionalista que nos propone Healey, en sus formas socio- constructivistas, pero tener presentes estos debates ayuda a focalizar la atención en estas interacciones.

\section{Gobernanza Ambiental}

Co-gestión de bienes comunes construir relaciones de confianza y colaboración mutua estado-poblaciones locales

Las formas de democracia deliberativa implican la posibilidad de manejar tiempos adecuados, involucrar a una amplia gama de actores, comunicar en lenguajes accesibles las complejidades de los temas en cuestión, promoviendo modalidades de aprendizaje colectivas de los mismos. Para luego llegar a decisiones, vinculantes, o con algún nivel de garantía mayor a una simple consulta, sobre la incidencia que este trabajo representa para los actores que dedican su tiempo a estos procesos (Goñi et alt, 2013).

En este sentido, podemos decir que en América Latina estamos transitando una fase de intentos de democracia participativa, en materia de contribuir desde un diverso conjunto de actores en el armado de las políticas públicas ambientales, como señala Cristina Zurbbrigen (Zurbriggen, 2011). Se suma la constatación que siempre eran recogidas a modo de diagnóstico social o sugerencias y la petición de construir procesos que vayan desde la deliberación sobre los objetivos y propuestas, hasta modalidades de seguimiento activo de la implementación y posibles figuras de co- gestión que colaboren a reforzar espacios existentes de decisión local, hacia figuras permanentes de gestión de los bienes comunes.

A nivel internacional existen lo que se llama, figuras o cuerpos de participación cívica intermedios que logran realizar una mediación operativa permanente, con recursos y capacidades suficientes en territorio, entre los gobiernos y las realidades locales como señalan Bacqué y Sintomer (2011). Esto no significa abandonar el camino de reforzar al tercer nivel de gobierno, con informaciones y formaciones adecuadas y pertinentes, incluso darles mayores recursos en la sensibilización y debate público de los temas que afectan cada territorio previo a la toma de decisiones. Por el contrario, refuerza la descentralización y la cercanía de la política, así como propone formas libres de intereses partidarios que se interesen y obtengan informaciones directas previniendo posibles casos de corrupción o mal gobierno (Benedikter y Michelotto, 2014)

Este último punto va de la mano con la idea de reforzar una ciudadanía ambiental activa que se capacite, tanto en las discusiones y monitoreo de los servicios ecosistémicos, como en la propuesta de proyectos activos de educación ambiental, sensibilización en temas de consumo sostenible, apoyar acciones y campañas, así como para desarrollar formas de auto-organización en la gestión de los recursos y la innovación a través de nuevas tecnologías menos invasivas. Existen varios ejemplos, como los citados por Kernay, Berkes, Charles, Pinkerton y Viber (2007), en ecosistemas costeros, que es uno de los ecosistemas con mayor presión a nivel mundial por el constante crecimiento de su población y la urbanización turística que duplica la presión y el equilibrio de los mismos.

A partir de un abordaje desde las relaciones internacionales, podemos decir que en la actualidad existe una (re) conceptualización sobre la gobernanza como una forma de gobierno que no pasa por la acción aislada de una élite político-administrativa relativamente homogénea y centralizada, sino por la adopción de formas de coordinación a distintos niveles, y multiactoral (Ruano de la Fuente, 2002), la cual puede operar como una síntesis entre 
legitimidad y gobernabilidad de las acciones a tomar en las temáticas ambientales. Esta nueva concepción de la gobernanza ambiental a nivel mundial puede consolidar una institucionalidad nacional a partir de marcos supranacionales o meta-reguladores, en donde el Estado no devenga en un "mega" actor o un interventor que garantice cambios de fondo, sino una figura de Estado moderadamente regulador capaz de instalarse en un espacio de "geometría variable" (Svampa, 2013). Esto es, reforzar las modalidades que habiliten las tomas de decisión y acción dentro de un esquema multiactoral de fragmentación de la sociedad civil en diversos movimientos, grupos, ONG y otro tipo de actores. El hecho de pensar a estas nuevas formas que restituyan protagonismo a los actores más débiles económicamente es fundamental, visto que el avance y peso de capitales privados transnacionales y regionales en la economía nacional y local ha crecido a lo largo del ciclo neodesarrollista (Gudynas, 2009; Bértola et al., 2014 citado en Goñi et al., 2019).

El desafío aquí es en gran parte identificar quiénes son los sujetos con los que tomar decisiones, y qué modalidades activar que no sean ingenuas, o de decisión de pequeñas intervenciones, mitigaciones, sensibilización, mientras que los decisores de gran escala ya no están presentes físicamente en los países. Como señala Diego Piñeiro (2012), el land grabbing está generalizando una gestión a la distancia, en la que la definición del país y sus tierras son parte de un tablero de juego mayor, regional e internacional, y en donde los propietarios de los capitales no tienen presencia física, ni una cara real.

Esto implica un trabajo profundo de reflexión sobre las formas de gobernanza

multiescalar que debemos desarrollar con respecto a la gestión de nuestros bienes comunes naturales. En este contexto, nos enfrentamos a un nuevo escenario en donde el rol de los propietarios de las tierras, de quiénes las utilizan, así como de los emprendimientos económicos de distinta índole presentes en los territorios estén involucrados.

La importancia de la dimensión colaborativa o participativa, en la protección de los bienes naturales, ha tomado protagonismo sobre todo desde los estudios de Ellinor Ostrom en 1999 sobre las formas sociales de gestión de los bienes comunes. En la actualidad, estudios de referentes en la planificación y gestión ambiental como los ya mencionados Kernay y Berkes, indican como central la introducción de las comunidades locales. Pero para ello, insisten los autores, no se puede ignorar la necesidad de inversiones, de espacios habilitantes, donde la información de los temas lleguen en tiempo y forma a los actores, donde se ponga especial atención en crear capacidades locales, comprendiendo también las estratificaciones sociales y los conflictos latentes. Varias investigaciones han demostrado que a través de mecanismos de participación claros y vinculantes, las comunidades adoptan roles centrales en la implementación, monitoreo e incluso co-gestión de estos recursos (Kernay et al., 2007).

Si miramos el panorama internacional de experiencias de gobernanza deliberativa en temas ambientales, nos encontramos que por un lado, muchas de estas responden a procesos de pequeña escala en la gestión directa de los recursos naturales, trabajos con grupos que viven de la pesca, forestación, cultivos variados, entre otros.

Por otro lado, como decíamos anteriormente, se han creado sobre todo figuras de co- gestión relacionadas a unidades ambientales, cuencas, ríos, costas, ecosistemas terrestres diferenciados, bosques, montañas, entre otros. Por un tema de centralidad del recurso agua, los más desarrollados a nivel internacional se encuentran en este ámbito, por lo tanto tenemos por ejemplo, contratos de río, comités de cuenca, consejos de acuíferos, entre otros. Asimismo, otros dos temas han impulsado experiencias de este tipo; las reservas de biodiversidad que han creado grandes figuras de co-gestión de las áreas protegidas, parques naturales, reservas marinas, etc. y la tenencia de la tierra, en community land trust, cooperativas agrarias, u otras formas colaborativas que consideran la propiedad de la tierra como un derecho colectivo, no personalizable y necesariamente sujeto a un uso sin fines de lucro, que favorezca la soberanía alimentaria de los pueblos, y reduzca el hambre y las desigualdades.

La planificación colaborativa en cuanto campo interdisciplinario que nace de la necesidad de comunicar una disciplina compleja como el urbanismo, e involucrar una variada gama de actores esta proponiendo una gobernanza ambiental que incluya varios de estos aspectos.

Cambiar dirección no es sencillo, pero es fundamental, este es el mensaje que llega de los territorios latinoamericanos, cuando logran hacer sentir realmente su voz, entre tanta represión, violencia y asedio del sistema económico neodesarrollista contemporáneo. Si bien existe una clara conciencia de la dificultad en emprender nuevos modelos de desarrollo económico, se entiende hoy más que nunca que es indispensable, dados los altos índices de contaminación y desigualdades sociales, que las actuales formas de economía extractivista están produciendo, dejando al continente vaciado de sus recursos y con una deuda ambiental difícilmente sanable si no se invierte la marcha en los próximos decenios.

Referencias 
Allen A. (2001). UN-Habitat, Implementing the Habitat Agenda, Inresearch of Urban Sustainability, Development Planning Unit. Londres, UK: University College of London.

Almeida, M.A. y Muçouçah, P.S. (1991). Mutirão e Autogestão em São Paulo: uma Experiência de Construção de Casas Populares. Pólis, 4.

Amendola, A. (2010). Oltre la frontiera. La filosofia del diritto post-sovrano tra frammentazione e ricomposizione. En P. Nerhot, L'identità plurale della filosofia del diritto, Nápoles, Italia: Edizioni Scientifiche Italiane.

Arendt, H. (1997). Che cos'è la politica, Milán, Italia: Edizioni di Comunità.

Arnstein, S.R. (1969). A Ladder of Citizen Participation. Journal of the American Institute of Planners, 35, 216.

Bacqué, M.H. y Sintomer, Y. (2011). La démocratie participative: histoire et généalogie. Paris, Francia: LaDecouverte.

Bairle, S. (2003). The Porto Alegre Thermidor: Brazil's 'Participatory Budget' at the crossroads. Socialist Register, 39, 305-328.

Beck, U. (2010). Desigualdad social y cambio climático. En Mostafavi, M. \& Doherty, G. (Eds.). Ecological Urbanism. Harvard University Graduate School of Design/ Lars Muller Publisher, Cambridge( Mass.)/ Baden.

Benedikter, T. y Michelotto, P. (2014). Strumenti di partecipazione deliberativa e di democrazia diretta a livello comunale. Politis.

Bookchin, M. (2012). L'ecologia della libertà. Emergenza e dissoluzione della gerarchia, Milán, Italia: Elèuthera.

Brenner, N. (2014). Implosions/ Explosions. Towards a study of Planetary Urbanization. Berlín, Alemania: Ed. Jovis.

Caffentzis, G. (2012). In the desert of cities: notes on the occupy movement in the US. En: K. Khatib, M. Killjoy y M. McGuire (Eds.). We are many: Reflections on movement strategy from occupation to liberation (389-398). 0akland, USA: AK Press.

Capone, N. (2016). Del diritto d'uso civico e collettivo dei beni destinati al godimento dei diritti fondamentali. Politica del diritto, (4).

Cauduro, A. (2017). L'accesso al farmaco. Milán, Italia: Ledizioni.

Cellamare, C., Cognetti, F. y Goñi Mazzitelli, A. (coords.) (2018). Enabling Space. Tracce Urbane, (3). Recuperado de: http://ojs.uniroma1.it/index.php/TU

Dardot, P. y Laval, Ch. (2014). Commun. Essai sur la révolution au XXIe siècle, París, Francia: La Découverte.

Day, J. F. (2005). Gramsci is dead. Anarchist Currents in the Newest Social Movements. Londres, UK: Pluto Press.

De Sousa Santos, B. (2003). Democratizar a democracia. Os caminhos da democracia participativa. Río de Janeiro, Brasil: Civilização Brasileira.

De Sousa Santos, B. (2018). Conferencia inaugural del primer encuentro de la red Latinoamericana Senti Pensante, metodologías participativas y ciencias sociales. Recuperado de: https://redsentipensante.org

De Tullio, M.F. (2020). Uguaglianza sostanziale e nuove dimensioni della partecipazione política. Nápoles, Italia: Editoriale Scientifica.

Ferrajoli, F. (2013). Beni fondamentali. En: AA.VV., Tempo di beni comuni, Annali del- la Fondazione Basso, Roma: 
Ediesse.

Ferrajoli, L. (2012). Principia juris. Teoria del diritto e della democrazia, Tomo I, Roma-Bari.

Goñi, A., Festa, D., Giangrande, A., Angeloni, L. Troisi, R. (2013). Democrazia Emergente. La Stagione dei Bilanci Partecipativi a Roma e nel Lazio. Roma, Italia: Ed. Gangemi.

Goñi, A., Bisio, N., Chiglino, L. y Venegas, M. (2019). Informe Final Proceso de Participación al Plan Nacional para el Desarrollo Sostenible. CURE, UDELAR- MVOTMA.

Grossi, P. (1992). Il dominio e le cose. Percezioni medioevali e moderne dei diritti reali, Milán, Italia: Giuffrè.

Grossi, P. (2006). La proprietà e le proprietà nell'officina dello storico. Nápoles, Italia: Editoriale Scientifica. Gudynas, E. (2014). Derechos de la Naturaleza. Etica biocéntrica y políticas ambientales. Ed Creative Commons, Lima, Perú.

Haraway, D. (2019). Chthulucene, Sopravivvere su un pianetta infetto. Roma, Italia: Nero edizioni.

Hardin, G. (1998). Extensions of “The Tragedy of the Commons. Science, 280, 682-683.

Hardin, G. (1998). The tragedy of commons. Science, New Series. 162.

Hauben, M. (1995). Participatory Democracy From the 1960s and SDS into the Future On-line. Recuperado de: http://www.columbia.edu/ hauben/ronda2014/sds.txt

Hayden, T. (2012). Participatory Democracy: From the Port Huron Statement to Occupy Wall Street: On its fiftieth anniversary, the founding declaration of SDS echoes today in democracy movements around the world. The Nation, del 26 aprile 2012.

Healey, P. (1997). Collaborative Planning, shaping places in fragmented societies. UK:Ed. Palgrave.

Hobbes, Th. (2017). Leviatán, o la materia, forma y poder de una república eclesiástica y civil. Méjico D.F., Méjico: Fondo de Cultura Económica.

Jessop, B.(2002). Liberalism, Neoliberalism, and Urban Governance: a State-Theoretical Perspective. Antipode, 34 (3).

Kant, I . (2006). Idea de una historia universal con propósito cosmopolita. En I. Kant, Defensa de la ilustración (7392). Barcelona, España: Alba.

Kernay J., Berkes, F., Charles, A., Pinkerton, E. y Viber, M. (2007). The Role of Participatory Governance and Community-Based Management in Integrated Coastal and Ocean Management in Canada Journal, 35 (1).

Kioupkiolis, A. (2019). The Common and Counter-Hegemonic Politics Re-thinking social change. UK: Edinburgh University Press.

Latouche, S. (2007). La scommessa della decrescita. Milán, Italia: Ed. Feltrinelli.

Maddalena, P. (2019). La inattualità del disegno di legge sui beni comuni della Commissione Rodotà, del 24 gennaio. Recuperado de: https://www.attuarelacostituzione.it/2019/01/24/la-inattualita-del-disegno-di-legge-sui-benicomuni-della-commissione-rodota/

Mancilla, M. y Bodin, Ö. (2019). Participation in Multiple Decision Making Water Governance Forums in Brazil Enhances Actors' Perceived Level of Influence. Policy Studies Journal, 47 (1), 27-51. 
Mark Weber, J., Kopelman, S. y Messick, D. M. (2004). A Conceptual Review of Decision Making in Social Dilemmas: Applying a Logic of Appropriateness. Personality and social psychology review, 8 (3), 281-307. doi: $10.1207 /$ s15327957pspr0803_4.

Marotta, S. (2013). La via italiana ai beni comuni, in Aedon. Rivista di arti e diritto online 1.

Mattei, U. (2011). Beni comuni. Un manifestó. Roma-Bari: Laterza.

Mattei, U., Nader, L. (2010). Il saccheggio. Regime di legalità e trasformazioni globali. Milán, Italia: Bruno Mondadori.

Micciarelli, G.(2014). I beni comuni e la partecipazione democratica. Da "un altro modo di possedere" ad "un altro modo di governare". Jura Gentium, 1.

Micciarelli, G. (2017). Introduzione all'uso civico e collettivo urbano. Munus, 1.

Micciarelli G. (2018). Commoning. I beni comuni come nuove istituzioni, materiali per una teoria dell'autorganizzazione. Nápoles, Italia: Editoriale Scientifica.

Miller, J. (1987). Democracy in the Streets. New York, USA: Simon and Schuster.

Negri, A. y Hardt, M. (2017). Assembly, Oxford University Press.

Ostrom, E. (1990). Governing the commons. The Evolution of Institutions for Collective Action di Ostrom, Cambridge University Press.

Ostrom, E. (2010). Beyond Markets and States: Polycentric Governance of Complex Economic Systems. The American Economic Review, 100 (3), 641-672.

Piñeiro, D. (2012). Land grabbing: concentration and "foreignisation" of land in Uruguay. Canadian Journal of Development Studies. Revue canadienne d'études du développement, 33 (4), 471-489.

Rancière, J. (1995). La Mésentente. París, Francia: Éditions Galilée.

Rawls J. A. (1999). Theory of Justicem. Cambridge (Mass), USA: Harvard University press.

Rodotá, S. (2012). Il diritto di avere diritti, Roma-Bari: Laterza.

Rosanvallon, P. (1976). L'Age de l'autogestion, ou la Politique au poste de commandement. París, Francia :Seuil. Ruano de la Fuente, J. (2002). La gobernanza como forma de acción pública y como concepto analítico. VII Congreso Internacional del CLAD sobre la Reforma del Estado y de la Administración Pública. Lisboa, Portugal, 8-11 0ct. 2002.

Stavrides, S. (2014). Emerging commonspace as a challenge to the city of crisis. AA. VV Crisis-Scapes. Athens and beyond, Athen: Synthesi. Recuperado de: https://research.vu.nl/ws/portalfiles/portal/1339137/CrisisScapesConferenceBookWeb\%281\%29.pdf

Stockholm International Water Institute (2012). Report. Nutrir un mundo sediento: desafíos y oportunidades. Grupo de Investigación en Alimentación y Agricultura de las Naciones Unidas (FAO) y el International Water Management Institute. Recuperado de : www.siwi.org

Svampa, M. (2013). «Consenso de los Commodities» y lenguajes de valoración en América Latina. Nueva Sociedad, (244).

Viale, G. (2011). La Conversione Ecologica. There is no alternative. Rimini, Italia: Ed NDA Press.

Villasante, T. (2002). Reflexividades socio-práxicas. Esquemas metodológicos participativos. Madrid, España: IepalaCimas. 
Viveiros de Castro, E. (2013). La Mirada del Jaguar, introducción al perspectivismo amerindio. Buenos Aires, Argentina: Ed. Tinta Limón, ed. original 2008.

Zurbriggen, C. (2011). Gobernanza: una mirada desde América Latina. Governance: A view from Latin America. Perfiles latinoamericanos, 19 (38), 39-64. 